\title{
False-positive PCR results linked to administration of seasonal influenza vaccine
}

\author{
T. Curran, ${ }^{1}$ C. McCaughey, ${ }^{1}$ J. Ellis, ${ }^{2}$ S. J. Mitchell, ${ }^{1}$ S. A. Feeney, ${ }^{1}$ \\ A. P. Watt, ${ }^{1}$ F. Mitchell, ${ }^{1}$ D. Fairley, ${ }^{1}$ L. Crawford, ${ }^{1}$ J. McKenna ${ }^{1}$ \\ and P. V. Coyle ${ }^{1}$ \\ ${ }^{1}$ Regional Virus Laboratory, Microbiology Dept, Kelvin Building, Royal Victoria Hospital, \\ Belfast BT12 6BA, UK \\ ${ }^{2}$ Respiratory Virus Unit, Virus Reference Department, Microbiology Services, \\ Health Protection Agency, Colindale, London NW9 5HT, UK
}

Correspondence

T. Curran

tanya.curran@belfasttrust.

hscni.net

Received 31 October 2011

Accepted 15 November 2011

\section{INTRODUCTION}

High-throughput molecular testing is increasingly becoming the mainstay of clinical virology laboratories, with many laboratories solely employing amplification-based techniques for viral respiratory infection diagnosis. Many virology laboratories delivering molecular influenza diagnosis during the 2009-2010 pandemic, and in the following 2010-2011 influenza season, employed an automated and algorithmic approach to testing, facilitating faster and greater clinical specimen throughput.

During the 2009-2010 influenza pandemic the Regional Virus Laboratory, Belfast, rationalized screening of respiratory specimens from patients presenting with influenzalike illness (ILI) meeting the case definition to influenza A [matrix (M) gene], H1N1 (2009) [haemagglutinin (HA)

Abbreviations: GP, general practitioner; HA, haemagglutinin; ILI, influenzalike illness; M, matrix; NA, neuraminidase. gene] and the housekeeping gene RNase $\mathrm{P}$ (to determine specimen quality). During the winter of 2010-2011, a broader approach to initial influenza screening was required, due to the co-circulation of influenza with other respiratory viruses causing ILI. The 2010-2011 algorithm tested for influenza A (M gene), H1N1 (2009), influenza B, respiratory syncytial virus (RSV) and RNase P. Samples with discrepant results were further tested with a subtyping panel of realtime PCR assays targeting H1N1 (2009) neuraminidase (NA), seasonal H1N1 and H3N2 HA.

The vast majority of samples received from patients with ILI during the pandemic and the 2010-2011 influenza season tested positive for one influenza subtype. However in both seasons, a small number of specimens collected from community cases of ILI, were positive for both influenza A $\mathrm{H} 3 \mathrm{~N} 2$ and influenza $\mathrm{B}$ in addition to either seasonal H1N1 or H1N1 (2009) viruses, giving rise to concerns of specimen contamination during laboratory testing. The repeated 
amplification of the same target sequence, resulting in accumulation of amplified product within the laboratory environment, can be a major source of contamination in molecular testing, and multiple control methods are routinely employed to control this (Aslanzadeh, 2004; Borst et al., 2004; Hartley \& Rashtchian, 1993). However, further laboratory analysis of these samples and influenza vaccine, in addition to environmental swabbing of an influenza vaccination area, confirmed source contamination.

Here we report this novel cause of contamination and discuss its significance.

\section{METHODS}

Clinical samples. During January 2009 to February 2011, 11033 specimens were tested for influenza in the Regional Virus Laboratory, Belfast. Both combined nasal and throat swabs in addition to sputum samples in either Nuclisens lysis buffer (bioMérieux) in 2009-2010 or eNat medium (Copan) in 2010-2011 were received. Specimens were received from hospitals and general practitioner (GP) practices in Northern Ireland and also from the 29 GP practices participating in the Northern Ireland GP sentinel surveillance scheme.

Molecular analysis. Following nucleic acid extraction of $200 \mu \mathrm{l}$ sample using the QIAsymphony platform (Qiagen), specimens were tested by real-time Taqman PCR according to the seasonal testing algorithms. These assays included generic influenza A ( $\mathrm{M}$ gene), influenza B (HA gene), H1N1 (2009) (HA gene), seasonal H1N1 (HA gene) and H3N2 (HA gene) (Health Protection Agency, 2009a, d, e; van Elden et al., 2001). The $10 \mu$ PCRs consisted of $1 \times$ Superscript III Platinum One step qRT-PCR (Invitrogen) with $0.4 \mu \mathrm{M}$ primers, $0.4 \mu \mathrm{M}$ Taqman probe and $2 \mu \mathrm{l}$ extracted specimen. All real-time assays were carried out on a LightCycler 480 real-time machine (Roche) using the following cycling conditions: $50{ }^{\circ} \mathrm{C} 15 \mathrm{~min}, 95{ }^{\circ} \mathrm{C} 5 \mathrm{~min}$, followed by 45 cycles of $95{ }^{\circ} \mathrm{C} 10 \mathrm{~s}$ and $60{ }^{\circ} \mathrm{C} 1 \mathrm{~min}$. Positive and negative controls were included in each PCR assay run. Specimens with crossing threshold $\left(C_{\mathrm{t}}\right)$ values $\leqslant 40$ were reported as positive.

Specimens in which an unusual pattern of PCR positivity for multiple targets was observed were repeat tested. Any specimens with the same unusual pattern of positivity were selected for further investigation. Specimens were re-extracted, retested in all real-time PCR assays in addition to two H5N1 specific assays (Health Protection Agency, 2009b, c; WHO, 2007) and underwent sequence analysis.

Detection of RNA in influenza vaccine. Trivalent 2010 vaccine from two manufacturers (Wyeth and Sanofi Pasteur), were obtained from the hospital Pharmacy and Occupational Health departments. Both trivalent vaccines contained an A/California/7/2009 (H1N1)-like virus, an A/Perth/16/2009 (H3N2)-like virus and a B/Brisbane/60/ 2008-like virus as recommended by the World Health Organization.

Two hundred and fifty microlitres of each were extracted using the QIAsymphony platform (Qiagen) and subsequently tested for influenza A and B viral RNA.

Sequencing. Partial sequencing of the $M$ gene was carried out on two of the specimens (14710/09 and 12821/10) with unusual patterns of PCR positivity, in addition to both vaccines, using the BigDye Terminator v3.1 Cycle sequencing kit (Applied Biosystems). Primers targeting a $153 \mathrm{bp}$ region were used: forward primer $5^{\prime}$-CTTGATATTGTGGATTCTTGA- $3^{\prime}$ and reverse primer $5^{\prime}$-ATCGTCAACATCCACAGCA-3' (primer positions 813-833 and 948-966 respectively on the M gene: GenBank accession number CY009317). Products were purified with the Montage Seq 96 sequencing reaction cleanup kit (Millipore) prior to sequencing on a 3100 capillary sequencer (Applied Biosystems). Sequences were analysed for homology using NCBI BLAST analysis. Additional PCR assays (not shown) targeting longer regions of the influenza $\mathrm{M}$ gene failed to generate products for sequencing.

The other three specimens were referred to the Respiratory Virus Unit, Health Protection Agency, Colindale, for further sequence analysis. Viral RNA was extracted from specimens using the NucliSens easyMag system (bioMérieux). Viral RNA was amplified using primers targeting the influenza A M gene and the amplicons were directly sequenced using the PCR primers AMF Forward (5'-GAGTCTTCTAACMGAGGTCGAAACGTA-3', nucleotides 17-43 in CY009317) and AMR Reverse (5'-GGGCACGGTGAGCGTRAA-3', nucleotides 204222) (Ellis \& Curran, 2011). PCR clean-up and sequencing reactions were performed by the Genomic Services Unit at the Department for Bioanalysis and Horizon Technologies, Health Protection Agency. PCR amplicons were prepared for sequencing by using Ampure Magnetic beads on the Biomek NxP robot (Beckman Coulter). Sequencing was performed as above, with clean-up of products with CleanSeq magnetic beads on the Biomek robot and automated sequence detection on a 48capillary ABI 3730 genetic analyser.

Environmental swabbing. Thirty-eight environmental swabs were taken in October 2010 in a Belfast hospital Occupational Health vaccination area following a single session where 123 health care workers had received influenza trivalent vaccine. All swabs were premoistened in tap water. A premoistened-only swab was also tested as a negative control. Swabs were set down on tables in the area in addition to streaking the swabs over various lengths (ranging from $2 \mathrm{~cm}$ to $1 \mathrm{~m}$ ). Each surface was individually swabbed four times. Swabs were immediately placed in $500 \mu$ l Nuclisens lysis buffer, nucleic acid extracted and tested for influenza.

RNA surface survival time. An RNA survival experiment was undertaken to estimate the stability and durability of influenza vaccine RNA on surfaces. A 1 in 1000 dilution of the Wyeth vaccine was made in tap water, applied evenly using cotton wool onto a laminate flooring material and allowed to air dry, stored in a non-laboratory domestic setting. Over the subsequent 9 weeks, a swab of a $4 \mathrm{~cm}$ trail was taken at each of eight time points. These swabs were placed in Nuclisens lysis buffer, extracted and tested by real-time PCR as described.

\section{RESULTS}

\section{Cases}

During the period from June 2009 to February 2011, a total of 11033 specimens were tested for influenza A, 8187 of which were also tested for influenza B (a subset of samples was influenza $B$ tested due to rationalization of the 2009-2010 testing algorithm for hospital and community specimens). Nineteen per cent $(2105 / 11033)$ of specimens were positive for influenza A, of which $18 \%(385 / 2105)$ were received from sentinel influenza GP practices and $82 \%(1720 / 2105)$ from non-sentinel GP practices and hospitals. The prevalence of influenza B from 2009-2011 was $3 \%(227 / 8187)$, of which $14 \%(32 / 227)$ were received from sentinel influenza GP practices and $86 \%(195 / 227)$ from non-sentinel GP practices and hospitals. Six specimens were positive for both H1N1 (2009) and influenza B.

During 2009-2011, an unusual pattern of influenza A H1N1 (2009), H3N2, seasonal H1N1 and influenza B virus detection 
by PCR was observed in five specimens, which were reextracted and repeat tested (two H5N1 real-time assays were also included in this repeat testing). The results are shown in Table 1. Four specimens collected and tested in 2009 were positive for seasonal $\mathrm{H} 1 \mathrm{~N} 1, \mathrm{H} 3 \mathrm{~N} 2$ and influenza $\mathrm{B}$, but negative for H1N1 (2009) and H5N1 viruses. A fifth specimen collected and tested in 2010 was positive for H1N1 (2009), $\mathrm{H} 3 \mathrm{~N} 2$ and influenza $\mathrm{B}$ viruses. This specimen was negative for seasonal $\mathrm{H} 1 \mathrm{~N} 1$ and $\mathrm{H} 5 \mathrm{~N} 1$ viruses. The age of the patients ranged from 16 to 76 years, with a $3: 2$ ratio of women to men. The 2009 specimens were from patients attending two different GP practices. Specimens 13584/09 and 13810/09 were taken 1 day apart in September 2009 in GP practice 1. Specimens 14136/09 and 14710/10 were taken 7 days apart the following month in GP practice 2. Specimen 12821/10 was taken in September 2010 in GP practice 3. None of the five patients had received that year's influenza vaccine prior to or on the day of sampling. There was no social or geographical link between the cases. All specimens had been taken in areas in the practices where both clinical sampling and influenza vaccination were regularly carried out.

\section{Detection of RNA in influenza vaccine}

Both 2010 trivalent vaccines were extracted and tested in all influenza Taqman real-time PCR assays. Influenza RNA from both trivalent vaccines was detected in all of the realtime PCR assays except those for detection of seasonal H1N1 and H5N1. $C_{t}$ values ranging from 11.79 to 13.79 were observed, representing a significant load, in the order of an estimated $10^{10}$ copies $\mathrm{ml}^{-1}$ of target (based on general extrapolation of $C_{\mathrm{t}} 28$ equivalent to $10^{5}$ copies $\mathrm{ml}^{-1}$ and a $\log$ value equatable to $3.32 C_{\mathrm{t}}$ ).

\section{Sequencing}

Amplicons from PCR targeting part of the $\mathrm{M}$ gene of specimens $14710 / 09$ and $12821 / 10$ in addition to both trivalent influenza vaccines were sequenced.
BLAST analysis on NCBI showed the $\mathrm{M}$ gene partial sequences from these specimens to have $100 \%$ homology to the M gene sequence of A/Puerto Rico/8/1934. Sequence analysis of PCR products generated following amplification of a different region of the influenza $\mathrm{A} M$ gene from specimens 13584/09, 13810/09 and 14136/09 generated similar results (Fig. 1).

\section{Environmental swabbing}

Environmental swabs from the occupational health vaccination area were tested and the results are shown in Table 2. Influenza RNA was detectable in all swabs in all assays except those for seasonal H1N1 and H5N1.

\section{RNA surface survival time}

Eight swabs corresponding to eight different time points were taken of diluted $(1: 1000)$ vaccine that had been applied and air-dried onto a piece of laminate floor material. The results are shown in Table 3. Diluted vaccine RNA remained relatively static over all time points, and was still detectable at day 66 .

\section{DISCUSSION}

The 2009 influenza pandemic was the first pandemic to occur since the advent of molecular techniques (McCaughey, 2010). This molecular approach to testing has allowed the introduction of and reliance on a wide range of rapid and sensitive virology assays. However, PCR contamination is often a consequence of amplicon-to-specimen and occasionally specimen-to-specimen contamination within the laboratory (Speers, 2006).

Five specimens collected and tested during 2009 and 2010 demonstrated unusual patterns of influenza virus RNA positivity; the 2009 specimens were positive for seasonal $\mathrm{H} 1 \mathrm{~N} 1, \mathrm{H} 3 \mathrm{~N} 2$ and influenza $\mathrm{B}$ but negative for H1N1

Table 1. Real-time PCR results of five cases tested 2009-2010

\begin{tabular}{|lccccc|}
\hline Virus (gene target) & \multicolumn{5}{c|}{$\boldsymbol{C}_{\mathbf{t}}$ values $^{*}$} \\
\cline { 2 - 6 } & $\mathbf{1 3 5 8 4 / 0 9 \dagger}$ & $\mathbf{1 3 8 1 0 / 0 9} \dagger$ & $\mathbf{1 4 1 3 6 / 0 9 \dagger}$ & $\mathbf{1 4 7 1 0 / 0 9 \dagger}$ & $\mathbf{1 2 8 2 1 / 1 0 \ddagger}$ \\
\hline Influenza A (M) & 34.20 & 33.97 & 32.13 & 36.89 & 35.07 \\
Influenza B (HA) & 35.67 & 35.83 & 39.18 & 37.17 & 31.25 \\
H1N1 (2009) (HA) & - & - & - & - & 36.28 \\
H1N1 (2009) (NA) & - & - & - & - & 34.02 \\
Seasonal H1N1 (HA) & 33.05 & 32.79 & 32.81 & 37.29 & - \\
H3N2 (HA) & 34.24 & 34.12 & 34.48 & 37.84 & 33.96 \\
H5N1 (HA)\$ & - & - & - & - & - \\
\hline
\end{tabular}

*-, Negative.

$\dagger 2009$ specimens.

$\ddagger 2010$ specimen.

$\S$ Combined results for both $\mathrm{H} 5 \mathrm{~N} 1$ assays. 
(a)

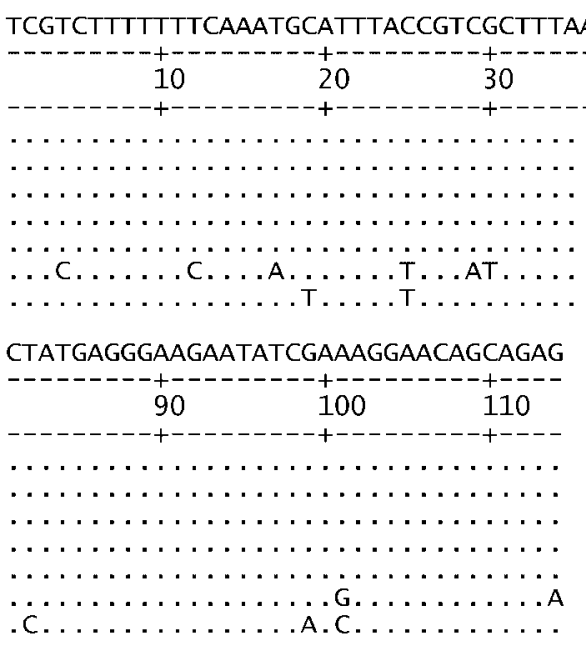

Consensus 0

A/Puerto Rico/8/1934 SP Vaccine wyeth Vaccine $14710 / 09$

$12821 / 10$

A/Brisbane/59/2007 A/California/07/2009

Consensus

(b)

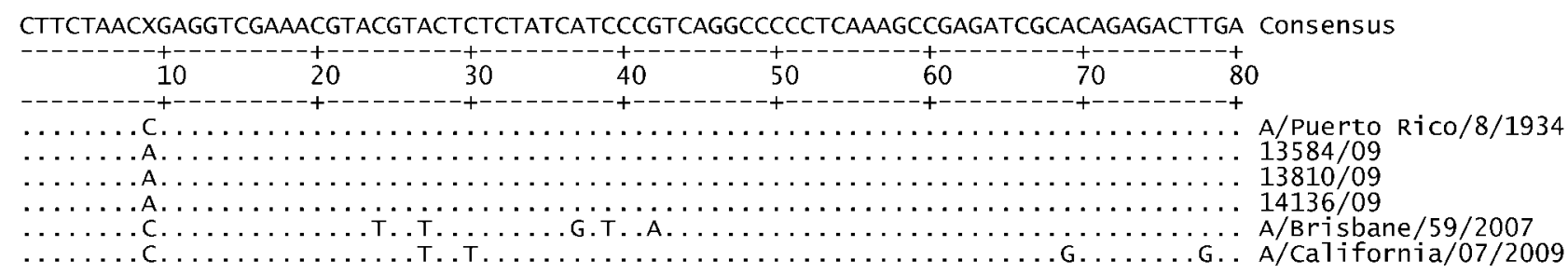

AGATGTCTTTGCAGGGAAGAACACCGATCTTGAGGTTCTCATGGAATGGCTAAAGACAAGACCAATCCTGTCACCTCTGA Consensus

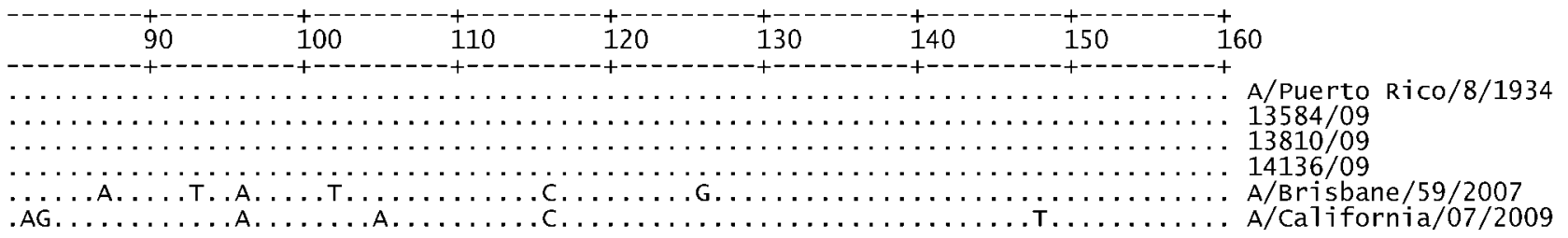

CTAAGGGGATTTTAGGATTTGTGTTCACGCTCACCGTGCCC

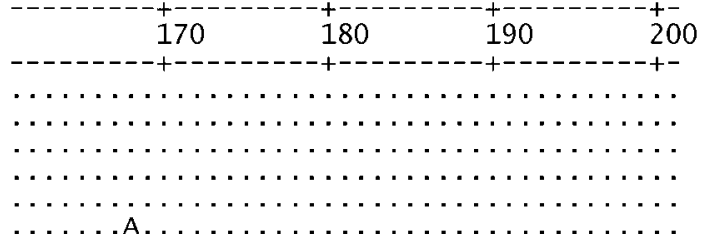

A/Puerto Rico/8/1934
SP Vaccine
Wyeth Vaccine
$14710 / 09$
$12821 / 10$
A/Brisbane/59/2007
A/California/07/2009

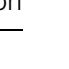


Table 2. Real-time PCR results of environmental swabbing of occupational health vaccination area

\begin{tabular}{|c|c|c|c|c|c|c|c|c|}
\hline \multirow[t]{2}{*}{ Swab } & \multirow[t]{2}{*}{ Description } & \multicolumn{7}{|c|}{$C_{\mathrm{t}}$ values $^{*}$} \\
\hline & & $\begin{array}{c}\text { Infuenza A } \\
(\mathrm{M})\end{array}$ & $\begin{array}{c}\text { Influenza B } \\
\text { (HA) }\end{array}$ & $\begin{array}{c}\text { H1N1 (2009) } \\
\text { (HA) }\end{array}$ & $\begin{array}{l}\text { H1N1 (2009) } \\
\text { (NA) }\end{array}$ & $\begin{array}{c}\text { Seasonal H1N1 } \\
\text { (HA) }\end{array}$ & $\begin{array}{c}\text { H3N2 } \\
\text { (HA) }\end{array}$ & $\begin{array}{c}\mathrm{H} 5 \mathrm{~N} 1 \dagger \\
\text { (HA) }\end{array}$ \\
\hline 1 & Set down Table $1 \ddagger$ & $36.42 \pm 0.67$ & $36.36 \pm 0.72$ & $36.72 \pm 1.77$ & $36.71 \pm 1.59$ & - & $38.67 \pm 2.00$ & - \\
\hline 2 & $2 \mathrm{~cm}$ trail Table $1 \S$ & $34.56 \pm 0.82$ & $32.36 \pm 1.60$ & $34.23 \pm 1.36$ & $33.81 \pm 0.79$ & - & $35.17 \pm 1.19$ & - \\
\hline 3 & $10 \mathrm{~cm}$ trail Table $1 \S$ & $35.46 \pm 0.73$ & $33.19 \pm 1.04$ & $35.38 \pm 0.82$ & $34.85 \pm 0.91$ & - & $35.79 \pm 0.86$ & - \\
\hline 4 & $1 \mathrm{~m}$ trail Table $1 \S$ & $33.43 \pm 2.09$ & $31.28 \pm 2.76$ & $33.22 \pm 2.65$ & $32.46 \pm 2.51$ & - & $33.66 \pm 2.52$ & - \\
\hline 5 & $2 \mathrm{~cm}$ trail chair $\$$ & $36.97 \pm 0.35$ & $36.58 \pm 1.37$ & $37.61 \pm 1.26$ & $38.99 \pm 0.06$ & - & $39.76 \pm 1.76$ & - \\
\hline 6 & $20 \mathrm{~cm}$ trail Table $2 \S$ & $33.56 \pm 2.34$ & $30.80 \pm 2.81$ & $32.96 \pm 2.48$ & $32.40 \pm 2.60$ & - & $33.15 \pm 3.18$ & - \\
\hline 7 & Floorll & $36.11 \pm 1.29$ & $37.28 \pm 3.24$ & $37.42 \pm 2.14$ & $37.18 \pm 2.29$ & - & $38.12 \pm 2.13$ & - \\
\hline 8 & Tables 2 and $3 \|$ & $33.86 \pm 2.92$ & $30.29 \pm 2.82$ & $32.41 \pm 2.31$ & $32.32 \pm 2.79$ & - & $34.90 \pm 4.48$ & - \\
\hline 9 & Extensive Tables 1-39 & $31.71 \pm 3.41$ & $29.09 \pm 3.66$ & $31.04 \pm 3.19$ & $30.76 \pm 3.29$ & - & $31.86 \pm 3.55$ & - \\
\hline 10 & Vaccinated arm\# & $30.08 \pm 2.16$ & $27.12 \pm 1.98$ & $29.52 \pm 2.13$ & $29.10 \pm 1.80$ & - & $30.19 \pm 1.87$ & - \\
\hline 11 & Negative control ${ }^{\star \star}$ & - & - & - & - & - & - & - \\
\hline
\end{tabular}

${ }^{*}$ Data are means \pm SD of four individual swabs. - , Negative.

$\dagger$ Combined result for both $\mathrm{H} 5 \mathrm{~N} 1$ assays.

$¥$ Swabs set down on Table 1 for a few seconds.

$\$$ Swabs moved $2 \mathrm{~cm}, 10 \mathrm{~cm}, 20 \mathrm{~cm}$ or $1 \mathrm{~m}$ along Table 1 , Table 2 or chair.

IISwabs trailed along floor or Tables 2 and 3.

SSwabs vigorously trailed backwards and forwards for several metres along Table 1, Table 2 and Table 3.

\#Skin swabs of vaccinated arm taken immediately post-vaccination.

${ }^{*}$ Swab moistened with same nuclease-free water as used to premoisten each swab used prior to sampling.

longer regions of the $\mathrm{M}$ gene proved unsuccessful, which may reflect fragmentation of the vaccine RNA.

The most credible explanation of these results is carryover or detection of vaccine RNA. A recent publication described the contamination of diagnostic swabs in horses who had previously received influenza vaccination (Diallo et al., 2011). While this has not previously been reported for inactivated human influenza vaccines, recipients of intranasal live attenuated vaccine can shed influenza RNA for up to 3 weeks post vaccination and give rise to positive results when tested by PCR (Freed et al., 2007). In addition, contamination of specimens with pertussis vaccine has been documented (Leber et al., 2010). Surprisingly high loads (in the order of $10^{10}$ copies $\mathrm{ml}^{-1}$ ) were detectable in both trivalent vaccines. The effect of such high RNA loads in these influenza vaccines has not been documented in the literature. Single-stranded H5N1 RNA has been shown to stimulate Toll-like receptors, increasing the efficiency of antigen presentation (Geeraedts et al., 2008). Therefore vaccine RNA may have an important role in immunogenicity and efficacy of the vaccine. However the presence of such high concentrations of RNA may contribute to the well-documented systemic side effects associated with influenza vaccine, due to cytokine production initiated by the vaccine RNA (Julkunen et al., 2000).

Table 3. Vaccine RNA surface survival time $\left(C_{t}\right.$ values $)$

\begin{tabular}{|c|c|c|c|c|c|c|c|c|}
\hline \multirow[t]{2}{*}{ Assay } & \multicolumn{8}{|c|}{$C_{\mathrm{t}}$ values $^{\star}$} \\
\hline & $1 \mathrm{~h}$ & $10.5 \mathrm{~h}$ & $24 \mathrm{~h}$ & 1.5 days & 3 days & 9 days & 20 days & 66 days \\
\hline Influenza A (M) & 36.83 & 35.69 & 36.20 & 35.35 & 35.44 & 35.57 & 34.79 & - \\
\hline Influenza B (HA) & 37.54 & 35.64 & 37.01 & 35.98 & 35.30 & - & 35.13 & - \\
\hline H1N1 (2009) (HA) & 39.06 & 35.38 & 35.79 & 34.36 & 34.72 & - & 35.37 & 36.77 \\
\hline H1N1 (2009) (NA) & 38.17 & - & 37.44 & 37.51 & 37.45 & - & - & 35.82 \\
\hline Seasonal H1N1 (HA) & - & - & - & - & - & - & - & - \\
\hline H3N2 (HA) & $>40$ & 37.43 & 38.18 & 36.83 & 35.86 & - & 37.07 & 37.75 \\
\hline $\mathrm{H} 5 \mathrm{~N} 1 \dagger(\mathrm{HA})$ & - & - & - & - & - & - & - & - \\
\hline
\end{tabular}

*-, Negative.

$\dagger$ Combined result for both H5N1 PCR assays. 
The level of RNA in these vaccines clearly has potential to generate environmental contamination. Specimens from all five cases came from GP practices that routinely used the same treatment room for clinical sampling and influenza vaccination. The fact that H1N1 (2009), H3N2 and influenza B (but not seasonal H1N1) RNA was detected in the environmental swabs collected during 2010 from the occupational health vaccination area and the ability to detect vaccine influenza RNA for at least 66 days indicates a significant risk for environmental contamination and subsequent contamination of clinical specimens collected from such locations.

Previous studies have documented the potential risk of false-positive nucleic acid amplification test results when specimens are taken in an area that is contaminated with target DNA (Meader et al., 2008). Therefore it is surprising that this phenomenon appears not to have been recognized for influenza previously. Possibly individual laboratories dismiss these types of results as erroneous because multiple targets are positive. It is important to realize early that vaccine contamination is a potential explanation, especially where there are multiple influenza virus positive results (H1N1, H3N2 and influenza B) in the same specimen. In addition, laboratories employing algorithmic approaches to testing are unlikely to recognize vaccine contamination, leading to incorrect clinical diagnoses, unnecessary laboratory work and unsound epidemiological conclusions.

Controlling this type of contamination would involve a few simple measures. Ideally, separate areas should exist for vaccination and clinical sampling. Staff administering vaccine should wear protective clothing (aprons and gloves) and should not leave the vaccination area. Opened swabs should not be set on surfaces either before or after swabbing. Dropped swabs should be repeated rather than sent to the laboratory. Surfaces in treatment rooms should be regularly cleaned after vaccine use. Previous studies have shown that the use of common household cleaning agents such as $1 \%$ bleach, $10 \%$ malt vinegar and antiviral wipes inactivate virus but have little effect on genome number (Greatorex et al., 2010). Products specifically validated to control contamination in laboratories are effective in eliminating amplicon contamination (double-stranded DNA) but may have little effect on single-stranded RNA. Therefore care needs to be taken to ensure that these approaches and products are effective in inactivating vaccine RNA.

All lines of evidence, including the vaccine mirroring pattern of false positivity, the detection of A/Puerto Rico/8/ 1934 reassortant sequence, the high RNA load in the vaccine, the environmental contamination and the stable persistence of the RNA on surfaces, point to source contamination with vaccine strain RNA. The use of trivalent influenza vaccines in the same area as clinical sampling can result in vaccine contamination of specimens and give rise to erroneous laboratory results.

\section{ACKNOWLEDGEMENTS}

The authors would like to thank staff at the Health Protection Agency, in particular Richard Allan in the Respiratory Virus Unit, for technical support.

\section{REFERENCES}

Aslanzadeh, J. (2004). Preventing PCR amplification carryover contamination in a clinical laboratory. Ann Clin Lab Sci 34, 389-396.

Borst, A., Box, A. T. \& Fluit, A. C. (2004). False-positive results and contamination in nucleic acid amplification assays: suggestions for a prevent and destroy strategy. Eur J Clin Microbiol Infect Dis 23, 289299.

Diallo, I. S., Read, A. J. \& Kirkland, P. D. (2011). Potential of vaccination to confound interpretation of real-time PCR results for equine influenza. Vet $\operatorname{Rec} 169,252$.

Ellis, J. S. \& Curran, M. D. (2011). Simultaneous molecular detection and confirmation of influenza AH5, with internal control. Methods Mol Biol 665, 161-181.

Freed, N. E., Myers, C. A., Russell, K. L., Walter, E. A., Irvine, M., Coon, R. G. \& Metzgar, D. (2007). Diagnostic discrimination of live attenuated influenza vaccine strains and community-acquired pathogenic strains in clinical samples. Mol Cell Probes 21, 103-110.

Geeraedts, F., Goutagny, N., Hornung, V., Severa, M., de Haan, A., Pool, J., Wilschut, J., Fitzgerald, K. A. \& Huckriede, A. (2008). Superior immunogenicity of inactivated whole virus H5N1 influenza vaccine is primarily controlled by toll like receptor signalling. PLoS Pathog 4, e1000138.

Greatorex, J. S., Page, R. F., Curran, M. D., Digard, P., Enstone, J. E., Wreghitt, T., Powell, P. P., Sexton, D. W., Vivancos, R. \& Nguyen-VanTam, J. S. (2010). Effectiveness of common household cleaning agents in reducing the viability of human influenza A/H1N1. PLoS ONE 5, e8987.

Hartley, J. L. \& Rashtchian, A. (1993). Dealing with contamination: enzymatic control of carryover contamination in PCR. PCR Methods Appl 3 (Suppl.), S10-S14.

Health Protection Agency (2009a). One step influenza multiplex real-time RT-PCR National Standard Method SOP VSOP 50.

Health Protection Agency (2009b). European Real-Time RT-PCR for Influenza A H5 viruses. National Standard Method VSOP 46 Issue 2 http://www.hpa-standardmethods.org.uk/pdf_sops.asp

Health Protection Agency (2009c). Real-time RT-PCR (Taqman) for influenza A H5 viruses. National Standard Method VSOP 41 Issue 5 http://www.hpa-standardmethods.org.uk/pdf_sops.asp

Health Protection Agency (2009d). Swine-lineage influenza A H1 specific fast real-time PCR. National Standard Method VSOP 29 Issue 5 http://www.hpa-standardmethods.org.uk/pdf_sops.asp

Health Protection Agency (2009e). Swine-lineage influenza A N1 fast real-time confirmatory PCR assay. National Standard Method VSOP 48 Issuel http://www.hpa-standardmethods.org.uk/pdf_sops.asp

Julkunen, I., Melén, K., Nyqvist, M., Pirhonen, J., Sareneva, T. \& Matikainen, S. (2000). Inflammatory responses in influenza A virus infection. Vaccine 19 (Suppl. 1), S32-S37.

Leber, A. \& other authors (2010). Vaccines shown to contain PCR-detectable DNA include Pentacel ${ }^{\mathbb{R}}$, Daptacel ${ }^{\mathbb{R}}$, and Adacel ${ }^{\mathbb{R}}$. Detection of Bordetella pertussis DNA in acellular vaccines and in environmental samples from pediatric physician offices. Interscience Conference on Antimicrobial Agents and Chemotherapy (ICAAC): Boston, USA. 
McCaughey, C. (2010). Influenza: a virus of our times. Ulster Med J 79, 46-51.

Meader, E., Waters, J. \& Sillis, M. (2008). Chlamydia trachomatis RNA in the environment: is there potential for false-positive nucleic acid amplification test results? Sex Transm Infect 84, 107-110.

Speers, D. J. (2006). Clinical applications of molecular biology for infectious diseases. Clin Biochem Rev 27, 39-51. van Elden, L. J. R., Nijhuis, M., Schipper, P., Schuurman, R. \& van Loon, A. M. (2001). Simultaneous detection of influenza viruses A and B using real-time quantitative PCR. J Clin Microbiol 39, 196-200.

WHO (2007). Recommendations and laboratory procedures for detection of avian influenza A (H5N1) viruses in specimens from suspected human cases. Available from http://www.who.int/influenza/ resources/documents/h5n1_laboratory_procedures/en/index.html 